\title{
Une recherche ouverte sur l'action publique ${ }^{\star}$
}

\author{
Marcel Jollivet* \\ Sociologie, Association NSS-Dialogues, Ladyss, CNRS, Université Paris Nanterre la Défense, Nanterre, France
}

En 1977, le ministère de l'Environnement de l'époque signe avec le CNRS une convention, au titre de laquelle il financera des recherches répondant à ses besoins de connaissances ${ }^{1}$. Il vient d'être créé (1970); il a un besoin tout particulier de s'appuyer sur la recherche pour concevoir et mettre en œuvre sa politique. Il se trouve que Robert Chabbal, le directeur général du CNRS, est en train d'opérer une véritable révolution dans la conception de la programmation scientifique de l'organisme. L'idée qui l'anime est d'y promouvoir une recherche «finalisée ». Et pour ce faire, il suscite la création de tout un ensemble de grands programmes fédérateurs autour de questions portant sur des objets à dimensions à la fois physiques, chimiques ou biologiques, techniques et sociales ${ }^{2}$ qui sont au cœur de - selon l'expression aujourd'hui consacrée - l'«agenda politique». Son souci est clairement de faire en sorte que la recherche éclaire la décision publique et, plus même,

\footnotetext{
ॠ Voir dans ce supplément le texte d'introduction de Marcel Jollivet.

*Auteur correspondant :

marcel.jollivet@parisnanterre.fr

${ }^{1}$ Ce texte s'appuie sur le rapport de l'association NSSDialogues intitulé «Expertise sur les formes d'ouverture entre disciplines et de partenariat en recherche dans les programmes de recherche du MEDDE », remis au ministère de l'Écologie, du Développement durable et de l'Énergie le 18/11/2014, 63 p. +37 p. (tableaux et figures). Ce document est consultable sur le site de l'association www.nss-dialogues.fr. Il résulte du travail de sept experts membres de l'association (Rémi Barré, Evelyne Brun, Marcel Jollivet, Yves Le Bars, Jean-Louis Martinand, Claude Millier, François Papy).

${ }^{2}$ Le captage de l'énergie solaire (PIRDES), la fabrication des médicaments (PIRMED), la conception de nouveaux matériaux (PIRMAT), les évolutions des océans (PIROCEAN) et, plus globalement, de l'environnement (PIREN) induites par les activités humaines; mais aussi les transformations de la ville (PIRVILLE) et du travail, de l'emploi et des modes de vie liées aux nouvelles technologies (PIRTTEM).
}

concourt à des réalisations techniques considérées comme d'intérêt collectif. C'est dans cet esprit qu'en réponse à la demande du ministère et moyennant contrepartie financière, le CNRS crée, en 1978, le Programme interdisciplinaire de recherche sur l'environnement (PIREN). Ce souci de mettre la recherche en phase avec des préoccupations politiques bouleverse littéralement le mode de fonctionnement et l'organisation du travail au sein du CNRS, qui se proclame haut et fort le bastion de la recherche «fondamentale », c'est-àdire d'une recherche qui, pour pouvoir se dire telle, doit précisément échapper aux influences de la société. De ce point de vue, il est significatif que, comme l'illustre l'intitulé du PIREN, tous les programmes alors créés se proclament «interdisciplinaires ». C'est la division du travail de recherche qui est la marque même de la recherche fondamentale que la recherche finalisée remet en cause. C'est bien à cette exigence que répondait la structure du PIREN. D'autres initiatives, bien antérieures, allaient dans le même sens (par exemple, le $\mathrm{PUCA}^{3}$ ). On peut dire que la fin des années 1970 constitue une période d'apogée pour la recherche finalisée. Qu'une telle place lui soit alors donnée au sein du CNRS est un symbole fort de la reconnaissance de sa légitimité sur le plan scientifique.

Mais, précisément parce qu'elle constituait une véritable entorse aux règles régissant la recherche dans la structure du CNRS, cette tentative de greffe déboucha sur un échec. Jugeant que les recherches lancées par le PIREN restaient trop proches de la recherche "académique» et trop éloignées de ses besoins, le ministère mit fin à la convention qui le liait

\footnotetext{
${ }^{3}$ Le Plan Urbanisme, construction, architecture est une structure de coordination interministérielle. Il a été créé en 1998, mais il s'inscrit dans la lignée du Plan Construction créé en 1971. Sa mission est à la fois de programmer de la recherche et de conduire des actions d'expérimentation et d'innovation dans ses domaines de compétence.
} 
au CNRS pour lancer ses propres recherches. C'est ainsi que le Service de la recherche, des études et $\mathrm{du}$ traitement de l'information sur l'environnement (SRETIE) a été amené à faire, à partir des années 1980, sa propre programmation scientifique. Cet exercice a été reconduit sans interruption jusqu'en 2016 par le Service de la recherche du ministère. C'est dire si ce dernier peut se réclamer d'une longue expérience d'une programmation scientifique conçue en appui à des politiques publiques ${ }^{4}$. La nécessité, qui a découlé de cet objectif, de créer un dispositif de recherche ministériel qui s'affranchisse des règles d'organisation et des pratiques canoniques de la recherche publique, oblige à une réflexion sur la façon de concevoir la recherche dans ses rapports à l'action. À quelles contraintes doit se plier la recherche telle qu'elle se fait quand elle doit aider à penser et à mettre en œuvre l'action publique ? Comment s'y prendre pour faire en sorte que les chercheurs sachent s'appuyer sur leurs compétences pour se mettre en mesure de faire face à cette requête et $\mathrm{y}$ répondre en respectant les règles d'une démarche scientifique? Sur quelle démarche de recherche cet exercice de réflexivité débouche-t-il? Et quelle «science» (quelle connaissance dotée de quelle véracité ?) produit-il ? ${ }^{5}$ Telles sont les questions, loin d'être anodines comme on le voit, face auxquelles le Service de la recherche du ministère mettait les chercheurs qu'il souhaitait mobiliser. Ses exigences s'inscrivent de deux façons dans la programmation qu'il conçut pour y répondre: dans les modalités d'organisation et de fonctionnement des programmes, et dans les règles fixées aux approches scientifiques à mettre en œuvre.

\footnotetext{
${ }^{4}$ L'esprit du PIREN survit toujours au sein du CNRS, à travers les «Observatoires Hommes-Milieux », mais les tribulations qu'il a subies traduisent bien la difficulté d'introduire cette démarche dans un dispositif régi par une division du travail de recherche fondée sur l'excellence disciplinaire. Ces difficultés annoncent celles auxquelles les programmes du ministère seront immanquablement confrontés en raison du fait qu'ils feront appel aux mêmes communautés scientifiques. Il n'en est que plus intéressant de voir en quoi l'ouverture à la recherche finalisée qu'ils offrent à ces dernières peut changer la donne. ${ }^{5}$ L'analyse qui suit a été faite à partir de deux types de sources : 1) des sources écrites: les textes d'appel à proposition de recherche des programmes sur la période récente (depuis 2006) et des documents retraçant les actions d'animation (leur importance est une des caractéristiques de ces programmes) initiées par le conseil scientifique; 2) des sources orales: entretiens systématiques avec les chargés de mission, entretiens complémentaires avec des présidents des conseils scientifiques et/ou avec des personnes ayant joué un rôle d'animation. Elle a porté sur les 22 programmes existant en 2013 ( $c f$. liste en fin de ce numéro).
}

\section{Des programmes nés d'une matrice politique}

Le souci que les programmes soient conçus en tant qu'outils d'appui à l'action publique s'inscrit de trois manières dans les modalités de leur organisation et de leur fonctionnement: dans leur structure (1), dans les circonstances de leur création et/ou dans leur évolution (2) et dans les modalités de restitution de leurs résultats auprès de personnes à qui ils sont destinés (3) :

(1) Le fait que le pilotage des programmes soit confié à deux organes (un comité d'organisation et un conseil scientifique) est un schéma classique. Mais trois traits originaux de ce bicéphalisme sont à souligner. Le premier a trait à la composition du comité d'organisation ${ }^{6}$. Elle est conçue de façon à faire véritablement de lui l'instance politique chargée de définir les attentes des opérateurs de l'État. Le second, c'est que le conseil scientifique a à concevoir sa programmation scientifique en partant de ces attentes. Cette tâche prend à contre-pied la façon habituelle de faire de la programmation scientifique, puisqu'il ne s'agit plus de la concevoir comme un prolongement d'un itinéraire d'acquisition de connaissances établies en vue de les approfondir, mais de chercher à baliser des itinéraires de recherche répondant à une forme de «commande» de la société, à travers la politique. Cette exigence est d'autant plus contraignante pour le conseil scientifique que, troisième originalité majeure, en cas de désaccord, c'est au comité d'organisation qu'il revient de décider des projets retenus et financés. Le conseil scientifique a à « traduire » (le mot est on ne peut plus approprié) ces choix en termes de recherche. Inversement, le comité d'organisation doit apprendre, à travers son dialogue avec le comité scientifique, à tenir compte des contraintes de la recherche dans sa façon de lui adresser ses demandes. En somme, cela fait près d'un demi-siècle qu'à travers ce dialogue, ces programmes travaillent à mettre, à leur façon et avec leurs objectifs propres, «la science en société».

(2) La création des programmes est intrinsèquement liée à l'agenda politique. Elle prend place dans les trois «moments » de l'action publique : la question à traiter vient de s'inscrire à l'agenda politique,

${ }^{6}$ Elle varie selon les programmes, mais elle répond à des principes communs en réunissant des représentants: des directions du ministère, des autres ministères concernés (dont celui de l'enseignement supérieur et de la recherche), des Agences (et autres structures) sur lesquelles le ministère s'appuie pour mener ses politiques, des acteurs majeurs (entreprises, associations, collectivités locales) impliqués dans la politique en cause. 
l'action publique est à concevoir; il existe déjà une législation ou une pratique de l'État dans le domaine, mais le bilan qui est tiré de l'expérience antérieure, ou une nouvelle conjoncture politique, conduisent à en changer le cours, l'action publique est à accompagner dans ses nouveaux développements; une action publique est en cours, mais fait question, il faut en évaluer les résultats ${ }^{7}$. Positionner la problématique du programme dans ce cadre général est le premier objectif des textes des appels à proposition de recherche (APR). Cela s'y traduit par une présentation des raisons de lancement des programmes d'où découle le fil conducteur des recherches à y mener ${ }^{8}$.

(3) Cet affichage des objectifs va de pair avec une identification claire des acteurs individuels ou collectifs, publics ou privés, auxquels le ministère destine les résultats des recherches. La démarche de recherche inclut les modalités de leur transmission à ceux-ci. Le cahier des charges imposé aux programmes comporte des clauses précises sur ce point. Le souci qui s'y exprime est de favoriser l'appropriation réelle des enseignements à tirer des recherches par les acteurs concernés pour qu'ils comprennent mieux les raisons et les objectifs de l'action ministérielle, la place qu'ils y occupent et le rôle qu'ils ont à y jouer, les éventuelles remises en cause auxquelles elle les contraint; il s'agit aussi de mieux les outiller pour y faire face. Cette optique se traduit notamment par le souci-sans l'écarter, cela va de soi-de sortir de la pure logique de la publication scientifique, avec les contraintes et les délais qu'elle implique, pour multiplier les opportunités d'échanges directs. D'où l'importance accordée à l'animation scientifique'.

\footnotetext{
${ }^{7}$ Aucun programme n'a comme objectif premier, au moment de sa création, l'évaluation d'une action publique déjà engagée; c'est en fait à travers les projets de recherche que cette évaluation se fait dans la mesure où, cas fréquent, ils portent sur des exemples d'applications de politiques en cours. Il arrive d'ailleurs que, dans le cas où une politique a déjà une certaine ancienneté, un programme entre dans une phase nouvelle de son développement en choisissant de s'orienter vers un bilan de son application. Il est clair aussi que, plus un programme est ancien, plus il y a de probabilités qu'il mêle les fonctions ou qu'il passe de l'une à l'autre.

${ }^{8} \mathrm{D}$ 'où le caractère inhabituel des attendus qui ouvrent les APR. On y retrouve toute la gamme des événements qui commandent l'action d'un ministère: mise en place d'une loi ou d'un dispositif interministériel, adaptation de la législation nationale aux directives européennes, application des engagements pris à la Conférence de Rio, mise en œuvre des conclusions de travaux de comités ministériels, etc.

${ }^{9}$ Celle-ci prend des formes multiples: publications ad hoc (synthèses, notes et fiches techniques, etc.), organisation de séminaires, colloques, etc.
}

\section{Des appels à propositions de recherche pragmatiques}

Fixer les règles des approches scientifiques à mettre en œuvre est la raison d'être des APR. Il leur revient de transcrire dans le champ scientifique la démarche générale de la programmation voulue par le ministère. Étant à la fois le produit du dialogue entre le Comité d'orientation et le Conseil scientifique, et l'outil de la mobilisation des communautés scientifiques, ils sont la pierre angulaire du dispositif de recherche. C'est à travers eux que s'exprime l'originalité scientifique des programmes, car leur conception et leur rédaction traduisent la façon dont sont recherchés les ajustements entre les ordres d'exigences respectives des deux organes en vue de lancer des recherches y répondant. Ils illustrent les innovations que cet exercice requiert. Ces innovations peuvent être regroupées sur deux axes majeurs: ouverture et diversité.

\section{Une ouverture à cinq facettes}

L'ouverture a cinq facettes. Quatre d'entre elles concernent l'éventail des disciplines auxquelles il est simultanément fait appel dans un programme donné. Elles se retrouvent dans tous les programmes, même si c'est à des degrés divers. La cinquième, moins systématique, porte sur la composition même de l'équipe de recherche.

La première a trait à la multiplicité des disciplines et des spécialités en sciences de la Terre et de la Vie rassemblées en vue de la saisie des objets et des phénomènes naturels impliqués dans la question de recherche (les composantes physiques du changement climatique, les composantes biologiques des écosystèmes, les perturbateurs endocriniens, les sols, l'eau, les pollutions...). Cela traduit le fait que l'action publique a davantage besoin d'une recherche recomposant ces objets pour avoir prise sur eux que d'une recherche les fragmentant toujours plus dans une démarche réductionniste tous azimuts (ce qui est la logique des programmes de recherche classiques ${ }^{10}$ ). Le conseil scientifique se doit donc de répertorier les disciplines nécessaires à cette connaissance aussi globale que possible, et d'inventer des problématiques originales visant à faire converger leurs démarches. Dans le cadrage global ainsi défini, ce qui est avant tout demandé à

\footnotetext{
${ }^{10}$ Cette exigence allant dans le sens d'une recomposition n'est pas exclusive d'un besoin d'approfondissement classique. L'originalité est que ce besoin est formulé à partir d'une construction de l'objet de recherche dans la perspective de l'action politique envisagée à son propos.
} 
chacun des chercheurs, c'est d'améliorer les connaissances qui sont indispensables à la conception de l'action publique et non pas - ou, en tout cas, pas nécessairement - celles qui sont considérées comme les plus pertinentes dans ce qui est considéré comme la dynamique «d'excellence» de sa discipline. Travailler dans cette perspective oblige les chercheurs à réfléchir aux rapports de leur discipline à l'action et à prendre conscience de leur diversité, de discipline à discipline.

Le second aspect de cette ouverture est la place qu'occupent dans la gamme des disciplines mobilisées les Sciences de l'ingénieur. Cela s'explique bien évidemment par le rôle médiateur que jouent ces disciplines entre connaissance et action. On retrouve là les finalités d'action que visent les recherches. Il s'agit de soumettre les compétences techniques existantes à l'épreuve des problèmes environnementaux qu'elles soulèvent et d'inventer les connaissances et les savoirfaire techniques alternatifs susceptibles d'être parties de leur solution.

Le troisième aspect de cette ouverture est l'appel fait aux Sciences de l'homme et de la société, au sens large $\mathrm{du}$ terme (y compris donc l'économie) ${ }^{11}$. Cette ouverture illustre le fait qu'en matière d'environnement, si une politique publique doit évidemment reposer sur la meilleure connaissance possible des phénomènes naturels qui l'imposent, elle doit aussi prendre en compte les causes et les conséquences économiques et sociales du problème posé pour être en mesure d'orienter et d'instrumenter de la façon la plus opportune possible l'action à mener ${ }^{12}$. La place croissante avec le temps qui est donnée à cet ensemble de disciplines dans pratiquement tous les programmes traduit la prise de conscience progressive de cette nécessité. Cela conduit d'ailleurs, de la même manière que pour les disciplines «naturalistes», à ouvrir l'éventail des disciplines à mobiliser et à développer des problématiques qui leur soient transversales. Le fait qu'il faille considérer dans son ensemble le champ du social auquel l'action publique va se trouver confrontée, fait également que les problématiques proposées aux chercheurs les contraignent à y situer leurs apports

\footnotetext{
${ }^{11} \mathrm{Y}$ compris aussi les sciences juridiques qui étaient absentes au début des programmes, mais qui ont fait une apparition marquée dans les derniers.

${ }^{12}$ D'où, pendant à la place occupée par les sciences de l'ingénierie technique, une propension à développer une démarche d'«ingénierie sociale» dans les programmes, à travers l'économie, le droit, les sciences de la gestion, mais aussi d'une façon plus générale. Cette tendance se retrouve dans la quête d'une ouverture vers les sciences participatives.
}

respectifs pour contribuer à une démarche ayant une cohérence d'ensemble. Et, comme pour les autres disciplines, à repenser la capacité - extrêmement variable, là aussi $1^{13}$ - de leur discipline à répondre aux exigences de l'action. Une des grandes évolutions des programmes est de passer d'une conception «instrumentale»des Sciences sociales à un accent mis sur leurs apports davantage en termes de réflexivité à la démarche de recherche.

Le quatrième aspect de cette ouverture découle du choix de mener de front, dans un même programme, les objectifs de connaissance des phénomènes naturels et des rapports sociaux qu'ils mettent en jeu. Ce choix met en évidence le fait que ce sont les facettes d'une même question, que les deux ordres de faits se conditionnent l'un l'autre. Cela pousse à l'approfondissement de leurs intersections, qui sont précisément les points chauds des politiques publiques. Cet approfondissement passe par un dialogue dans lequel Sciences de la nature et Sciences de la société sont amenées à élaborer des problématiques en miroir novatrices ouvrant la porte à une interdisciplinarité élargie, voire à une transdisciplinarité $^{14}$.

Ainsi, pour répondre à la double requête des caractéristiques propres des problèmes environnementaux et du rapport à l'action publique réformatrice qu'ils requièrent, les programmes ont été conduits à adopter une dynamique de recherche commune placée sous le signe, pour le moins du rapprochement et idéalement des décloisonnements, entre les disciplines. Ils s'appuient sur les disciplines déjà fédératrices (comme l'écologie, l'agronomie, la géographie, les Sciences de l'ingénieur...) pour amplifier les potentialités d'ouverture qu'elles contiennent; ils créent les conditions pour que d'autres hybridations se réalisent; ce faisant, ils offrent l'opportunité de renforcer des affinités déjà virtuelles, mais aussi d'inventer et de mettre en chantier des

\footnotetext{
${ }^{13}$ Les programmes ont conduit à mettre en évidence la tension existant dans les différentes disciplines entre une posture fondamentalement distanciée, critique, et une posture d'ingénierie sociale.

${ }^{14}$ On parle ici d'interdisciplinarité «élargie» pour la distinguer de celle qui est couramment pratiquée dans la recherche telle qu'elle se fait, qui consiste à mêler les connaissances de disciplines et de spécialités proches (interdisciplinarité «de proximité»). La forme la plus achevée de l'interdisciplinarité «élargie» est celle qui associe sciences de la nature et sciences de l'homme et de la société. Ce peut être aussi l'association d'une discipline technique (par exemple: l'informatique) et d'une discipline thématique (par exemple: la linguistique). La transdisciplinarité est ici prise au sens du partage de concepts entre des disciplines d'univers intellectuels différents (par exemple, les concepts de résilience et de territoire).
} 
Tableau 1. Les différentes formes de l'ouverture entre les disciplines selon la structure des APR.

\begin{tabular}{|c|c|}
\hline $\begin{array}{c}\text { Les trois structures des APR } \\
\text { (nombre de programmes rentrant dans le cas de figure } \\
\text { correspondant) }\end{array}$ & Les formes de l'ouverture entre disciplines \\
\hline $\begin{array}{l}\text { 1) Un seul volet (en l'occurrence en «sciences } \\
\text { de l'Homme et de la société») (3) }\end{array}$ & $\begin{array}{l}\text {-Pluridisciplinarité* } \\
\text {-Interdisciplinarité de proximité }\end{array}$ \\
\hline \multirow[t]{2}{*}{$\begin{array}{l}\text { 2) Deux volets autonomes (un «sciences de la Vie } \\
\text { et de la Terre/sciences de l'ingénieur» et un «sciences } \\
\text { de l'homme et de la société») (6) }\end{array}$} & $\begin{array}{l}\text { A) Sans ponts entre eux } \\
\text { Au niveau de l'APR } \\
\text { - Pluridisciplinarité } \\
\text { Au niveau de chacun des volets } \\
\text { - Pluridisciplinarité } \\
\text { - Interdisciplinarité de proximité }\end{array}$ \\
\hline & $\begin{array}{l}\text { B) Avec des approches transversales entre les deux volets } \\
\text { - Interdisciplinarité de proximité } \\
\text { - Interdisciplinarité élargie } \\
\text { - Transdisciplinarité } \\
\text { **** }\end{array}$ \\
\hline $\begin{array}{l}\text { 3) Une liste de thèmes transversaux aux SVT } \\
\text { et aux SHS (13) }\end{array}$ & $\begin{array}{l}\text { A) Sans incitations à l'association des disciplines } \\
\text { - Pluridisciplinarité } \\
\text { B) Avec incitations à l'association des disciplines } \\
\text { - Interdisciplinarité de proximité } \\
\text { - Interdisciplinarité élargie }\end{array}$ \\
\hline
\end{tabular}

* L'APR propose une simple juxtaposition des approches disciplinaires.

** L'APR souhaite des projets mettant en interaction des approches disciplinaires entre disciplines proches $\left(c f\right.$. note $\left.{ }^{14}\right)$.

*** L'APR souhaite des projets créant des interactions entre des disciplines qui n'en ont habituellement pas $\left(c f\right.$. note $\left.{ }^{14}\right)$.

**** Recours à des concepts communs $\left(c f\right.$. note $\left.{ }^{14}\right)$

recompositions remettant en cause les véritables fossés que la division du travail scientifique qui a actuellement cours a créés entre champs disciplinaires (en particulier entre les Sciences de la nature et les Sciences de l'homme et de la société). Considérés dans leur globalité, les APR offrent une vision d'ensemble des problématiques interdisciplinaires, tant de proximité qu'élargie que la recherche a à couvrir pour faire face à la question de l'environnement et du développement durable. Leurs structures générales illustrent trois types de démarches (cf. Tab. 1). Celles-ci traduisent à la fois la complexité propre à chacune des thématiques et l'état des pratiques de recherche des communautés qu'elles obligent à mobiliser. Autant de programmes, autant de voies ouvertes à des décompositions/recompositions des découpages disciplinaires établis et à la fécondité des questionnements.

Mais, expression du souci des conseils scientifiques de répondre à la mission qui leur était confiée en prenant à bras le corps les défis qu'elle pose à la recherche, les APR font appel à une forme d'ouverture qui va encore plus loin en ce qu'elle tire les ultimes conséquences de l'objectif d'appui aux politiques publiques: l'association à la recherche de personnes qui ne sont pas statutairement des chercheurs, en vue de réduire au maximum la discontinuité entre la recherche et l'action. Il peut s'agir d'agents territoriaux de l'administration, d'acteurs économiques, de membres d'associations. La nouveauté, les difficultés de mise en pratique, mais aussi les questions épistémologiques que pose la radicalité de cette piste de recherche (dite «participative »), font que ce n'est que tardivement qu'elle est avancée. Mais la pratique tend à se développer dans les dernières années. Elle va dans le même sens que l'appel croissant aux sciences de la société. Et surtout, la nécessité ressentie d'en venir à ce genre de pratique a fait qu'un programme spécifique (le programme REPERE ${ }^{15}$ ), visant à faire travailler ensemble des chercheurs et des membres d'associations, a fini par être lancé (en 2010). Cette quête d'une osmose entre le monde de la recherche et la société civile renvoie en fait à une évolution générale et en profondeur de la conception de l'action publique, autour de l'idée de démocratie participative (Tab. 2).

Cet ensemble d'ouvertures traduit un souci particulier de se donner une vision d'ensemble des composantes

\footnotetext{
${ }^{15}$ Le « Réseau d'échanges et de programmes sur le pilotage de la recherche et l'expertise» a comme objectif de «renouveler les relations entre sciences et sociétés en explorant les voies de la participation des associations portant les enjeux du développement durable à la recherche et à l'expertise.»
} 
Tableau 2. Le partenariat en recherche selon les programmes.

\begin{tabular}{|c|c|}
\hline $\begin{array}{c}\text { Cas de figure } \\
\text { (nombre de programmes rentrant dans le cas de figure } \\
\text { correspondant) }\end{array}$ & Commentaires et/ou exemples \\
\hline $\begin{array}{l}\text { La communauté scientifique associe déjà étroitement } \\
\text { connaissances et action. (5) }\end{array}$ & $\begin{array}{l}\text { Ingénieurs des eaux et forêts, ingénieurs du génie civil, } \\
\text { Centres techniques }\end{array}$ \\
\hline $\begin{array}{l}\text { Les éléments d'une communauté scientifique existent, mais } \\
\text { elle doit être créée. (1) }\end{array}$ & Écologie/biologie/médecine/laboratoires \\
\hline $\begin{array}{l}\text { L'affichage du partenariat en recherche en application du cahier } \\
\text { des charges se traduit par des degrés variables d'exigences dans } \\
\text { la coopération entre chercheurs et acteurs. (10) }\end{array}$ & $\begin{array}{l}\text { L'incitation à une coopération effective avec les acteurs de } \\
\text { tous genres, y compris les associations, est mentionnée, mais } \\
\text { va de la simple recommandation à une quasi-prescription. }\end{array}$ \\
\hline $\begin{array}{l}\text { Il est tout particulièrement dicté par la dimension locale de la } \\
\text { question. (5) }\end{array}$ & $\begin{array}{l}\text { Dimension territoriale de la gestion des ressources et des } \\
\text { milieux naturels. }\end{array}$ \\
\hline $\begin{array}{l}\text { L'association des chercheurs et des acteurs est l'objet même } \\
\text { de la démarche de recherche. (1) }\end{array}$ & $\begin{array}{l}\text { Associer chercheurs et militants associatifs dans une } \\
\text { recherche. }\end{array}$ \\
\hline
\end{tabular}

d'une politique publique, de façon à mieux en cibler les objectifs et les modalités les plus appropriées de réalisation. On peut résumer ce parti-pris en disant que les programmes font le choix, qui renvoie aux nécessités de l'action, de la complexité. Cela leur permet de tenir le plus grand compte des particularités de chaque question et de chaque situation. S'il est bien attendu de chacune des disciplines convoquées qu'elle s'appuie sur son corpus de connaissances, ce n'est pas pour simplement l'appliquer à un cas de figure nouveau, ni pour en tester une énième fois la robustesse en soi, mais pour le mettre à l'épreuve d'un questionnement décalé, s'interroger sur son apport heuristique dans une démarche d'ensemble qui déborde la matrice intellectuelle dont il est le produit. Plus que les connaissances elles-mêmes, à travers leur mobilisation, ce sont les compétences sur lesquelles elles reposent, et la capacité qu'elles donnent au chercheur de se livrer à un exercice de réflexivité original conditionné par un contexte et un objectif donnés, qui sont requises. C'est là que les connaissances apportées par les acteurs jouent un rôle stimulant. Cette exigence de plasticité, et de soumission à la diversité qui en découle, correspond à une nécessité lorsqu'il s'agit d'action. Mais elle oblige à inventer une démarche de recherche en mesure d'y répondre: celle précisément que se sont attelés à construire les programmes du ministère.

\section{Une nécessaire adaptation à l'objectif d'une recherche pour l'action}

Considérés dans leur ensemble, les vingt-deux programmes examinés illustrent de plusieurs façons leur ajustement aux besoins de connaissances scientifiques du ministère ( $c f$. Tab. 1). Leur diversité tient bien sûr en premier lieu à l'extrême diversité des thèmes qu'ils abordent. On y retrouve la multiplicité des facettes de la question d'un environnement et d'un développement durables. Mais elle tient aussi à deux autres ordres de facteurs tout aussi essentiels s'agissant d'une recherche en appui à l'action publique : des facteurs qui ont trait aux conditions générales d'une mise en œuvre d'une programmation scientifique, des facteurs particuliers liés à des politiques précises et renvoyant de ce fait au stade où en est l'action publique quand le programme de recherche qui lui est dédié est lancé.

La mise en œuvre d'une programmation scientifique dépend en premier lieu de l'état des connaissances dans l'ensemble des domaines de recherche correspondant aux questions que le conseil scientifique retient comme étant celles qu'il convient de privilégier. De ce point de vue, la gamme des situations est très diverse; elle va du cas de figure où, la question étant nouvelle, les connaissances de base sont nettement insuffisantes ou mal identifiées à celui où, au contraire, il s'agit de mobiliser des connaissances existantes pour en faire le point de départ d'approfondissements. Dans le premier cas, établir l'état des lieux des connaissances est un prérequis, le programme est d'une facture classique -à ceci près qu'il ouvre plus largement le champ d'investigation à couvrir. Le second cas se prête plus aux dépassements de connaissances déjà partiellement partagées et aux affinements des problématiques.

Mais dans tous les cas, il s'agit d'amener des communautés scientifiques à se rapprocher pour mettre en commun un objet ou une question de recherche. Là est l'enjeu principal pour les conseils scientifiques : trouver les forces de travail nécessaires à l'atteinte de leurs 
objectifs; et donc, trouver les moyens de les mobiliser. Les programmes cherchent à agir comme des aimants pour attirer ces forces et donner plus de poids aux problématiques qu'ils souhaitent voir prises en charge par les communautés scientifiques; la plupart des programmes ont l'ambition de créer les communautés scientifiques dont ils ont besoin. Là aussi, les situations sont très diverses. Tel programme s'adresse à une communauté existante dont il s'agit de faire évoluer les problématiques, ou qu'il s'agit d'élargir à d'autres composantes. Tel autre au contraire a comme première tâche de provoquer les rapprochements de base indispensables entre des communautés scientifiques qui s'ignorent, voire dont les rapports sont délicats à traiter (questions de préséance, de territoires, de divergences théoriques, etc.). Le succès de ces remises en cause des découpages qui organisent le travail scientifique dépend de l'attrait des problématiques proposées. Or, les décentrages des questionnements sur lesquels reposent les APR du fait de leur assujettissement à leur finalité d'appui aux politiques publiques les mettent en porte-à-faux par rapport aux problématiques qui ont cours dans les différents champs disciplinaires. Les comités scientifiques doivent donc trouver la cote la moins mal taillée possible pour susciter cet attrait tout en maintenant l'originalité de leurs démarches. Le dialogue n'est pas seulement entre le comité d'orientation et le conseil scientifique, mais aussi entre le conseil scientifique et les communautés scientifiques auxquelles il s'adresse. Tout un travail aussi invisible et méconnu qu'original s'accomplit à ce stade de conception des textes des APR (il se poursuit bien évidemment à travers les évaluations et les activités d'animation). Ce travail est à la fois socialement utile puisqu'il vise à mettre en prises réciproques, via l'action politique, la recherche sur la société et la société sur la recherche, et scientifiquement novateur puisqu'il ouvre le chantier visant à créer, à partir de la pratique, pragmatiquement, la démarche de recherche assurant cette mise en interface. Les programmes mettent en place, entre l'action publique instruite par la recherche et le travail scientifique ciblé sur l'action publique, des boucles qui sont censées respectivement se féconder.

Cet ajustement dynamique ressort bien de la comparaison entre les programmes. Le propre de chacun des programmes est de trouver le meilleur angle d'attaque possible compte tenu du cas de figure face auquel il se trouve. L'analyse de programmes d'une certaine ancienneté fait apparaître qu'il est même exigé d'eux de s'adapter aux évolutions d'une politique publique donnée. Dans la conception des programmes, une telle démarche en temps réel est tout à fait recevable dans la mesure où elle conduit à combiner évaluation de la phase passée et propositions pour la phase à venir. Dans la vie des programmes, cela se traduit par les évolutions des contenus des APR, voire par le souci de marquer - par une numérotation - le passage d'une phase à une autre. Revient au conseil scientifique le travail tout à fait inhabituel d'adapter les problématiques aux périodes de l'action publique et d'en assurer les évolutions tout en préservant la continuité nécessaire au travail de recherche. Ces programmes peuvent donc être un outil d'une grande souplesse pour le ministère tout en s'inscrivant dans une dynamique de recherche cohérente. Il s'agit toujours d'inventer une démarche de production d'une connaissance de portée générale, mais qui tire sa dynamique de l'action publique.

Cette grande diversité des programmes fait partie du caractère novateur de l'expérience de recherche dont il est ici question au même titre que les règles générales de l'exercice de programmation scientifique dont ils sont le produit. Les lignes générales de cette programmation lui donnent son originalité. La diversité qu'elles produisent lui donne sa richesse ${ }^{16}$. Certains des programmes s'inscrivent dans une culture scientifique et technique bien établie et la confortent en l'adaptant au contexte actuel; de ce fait, ils innovent peu en démarche. Ils sont minoritaires. Dans leur grande majorité, au contraire, les programmes jouent le jeu. Celui d'abord de prendre en charge les incertitudes de tous ordres face auxquelles l'action publique se trouve; et de s'astreindre, pour y répondre, à des contraintes prenant à contre-pied les règles habituelles de programmation scientifique. Mais aussi, ce faisant, celui de prendre également à contre-pied les habitudes de pensée qui régissent le fonctionnement social des communautés scientifiques; et d'inventer des formes de communication et d'échanges scientifiques pour les faire évoluer. Application d'un protocole commun, les programmes illustrent autant de façons circonstanciées de l'adapter. Ainsi, chacun d'eux contribue à une meilleure identification des caractéristiques propres de la recherche finalisée en tant que démarche scientifique. Et, par-là, à la compréhension des obstacles auxquels elle se heurte et des conditions de son développement.

\section{Conclusion}

Cette floraison de pratiques innovantes est soustendue par une ambition majeure qui n'est jamais énoncée, mais qui n'en est pas moins la raison d'être de la dualité des organes de gouvernance des programmes : il s'agit rien de moins que de sortir la recherche du fossé de

\footnotetext{
${ }^{16} \mathrm{La}$ diversité des programmes se retrouve dans - et est augmentée par - celle qui découle de leur mise en œuvre dans le cadre des projets de recherche qu'ils financent ( $c f$. dans cette publication le texte d'Agathe Dufour, Jean-Baptiste Narcy, Judith Raoul Duval «La recherche finalisée, une approche ouverte sur la singularité des terrains »).
} 
l'opposition entre «excellence» et «pertinence» qu'il est devenu habituel de mettre en avant pour faire état de la difficulté de concilier finalité et qualité de la recherche - quand ce n'est pas, plus profondément, pour établir une hiérarchie de valeur entre les formes de recherche placées sous le signe respectif de l'un et l'autre de ces deux critères. L'ambition de produire une recherche répondant aux attentes du ministère (donc «pertinente») ne peut évidemment pas faire l'impasse sur sa qualité (son "excellence»). Le cahier des charges commun à tous les appels à proposition de recherche est d'ailleurs explicitement bâti sur l'idée que non seulement les deux ordres de critères sont conciliables, mais qu'ils se renforcent l'un l'autre. Cet a priori est la pierre de touche des programmes et l'enjeu qui va être mis à l'épreuve dans leur montage.

Le croisement de ces deux ordres de critères crée en effet un double registre d'obligations : un qui concerne les conseils scientifiques, un qui concerne les équipes. Aux premiers, il revient de concevoir les appels à proposition de recherche comme des matrices où ces croisements puissent naître. C'est ce qu'ils font dans la plupart des programmes et cela se traduit par le caractère volontairement très général et ouvert du cadrage offert par les textes des appels aux équipes. Obligation est ainsi faite à ces dernières de s'emparer des croisements virtuels multiples qui leur sont offerts et de faire de leurs projets des bancs d'essai pour leur donner corps. Sur ces bases, conseils scientifiques et équipes ont à faire ensemble le travail de s'accorder sur ce que peuvent être les critères d' «excellence» d'une démarche scientifique abordant des questions de recherche " pertinentes » (c'est-à-dire qui répondent aux besoins de l'action publique) et non pas dictées par une logique en soi d'approfondissements disciplinaires. Cette ambition est bien, par nécessité compte tenu des objectifs des recherches, au cœur de ce travail, qui se produit dans l'enchevêtrement des boucles liant appel d'offres successifs, montage des projets, évaluations ex post et ex ante, animations, publications.

Mais, n'étant pas énoncée, elle n'est pas l'objet d'une réflexion. Ni même d'une simple perception de la part des acteurs qui, pourtant, la portent. Tout à leur tâche, souvent difficile en raison des contraintes inhabituelles auxquelles ils sont confrontés, ceux-ci s'efforcent en premier lieu de les accomplir du mieux possible. D'où leur pragmatisme foncier. Et de bon aloi: c'est de recherche en train de se faire qu'il s'agit. En outre, ils sont sous la pression légitime de l'ancrage ministériel des programmes. Il n'en demeure pas moins que l'acquisition de la légitimité scientifique de la recherche finalisée passe par cette indispensable réflexion sur les critères de scientificité qui correspondent à ce qu'elle est: un mariage entre la recherche et l'action.

Le nombre de programmes sur lesquels elle porte, leur diversité et le recul que donnent les trois décennies qu'elle a couverte font que cette expérience offre un matériau unique pour caractériser une démarche de recherche intégrant les contraintes de l'action publique dans ses objectifs d'acquisition de connaissances. Les analyses qui précèdent sont une contribution à cette caractérisation. Mais elles n'ont de sens que si elles sont un point de départ pour cette réflexion qui permettra de donner leurs fondements théoriques et épistémologiques aux pratiques qu'elles mettent en évidence. C'est à cette condition que la recherche finalisée sortira de la sorte d'inconsistance formelle dans laquelle la maintient le pragmatisme avec lequel on en use et qu'elle acquerra la «robustesse » nécessaire pour devenir l'outil dont notre époque a besoin pour que la recherche accompagne le processus démocratique ${ }^{17}$.

Citation de l'article : Jollivet M., 2017. Une recherche ouverte sur l'action publique. Nat. Sci. Soc. 25, S21-S28.

\footnotetext{
${ }^{17}$ Je remercie Yves Le Bars, Claude Millier et François Papy pour leur lecture vigilante qui m'a permis d'améliorer ce texte.
} 